\title{
A method to generate near real time UV-Index maps of Austria
}

\author{
B. Schallhart ${ }^{1}$, M. Blumthaler ${ }^{1}$, J. Schreder ${ }^{2}$, and J. Verdebout $^{3}$ \\ ${ }^{1}$ Division of Biomedical Physics, Innsbruck Medical University, Muellerstrasse 44, 6020 Innsbruck, Austria \\ ${ }^{2}$ CMS Ing.Dr.Schreder GmbH, Eggerstrasse 8, 6322 Kirchbichl, Austria \\ ${ }^{3}$ European Commission, Joint Research Centre, Institute for Health and Consumer Protection, Via E. Fermi 1, \\ 21020 Ispra (VA), Italy
}

Received: 20 December 2007 - Published in Atmos. Chem. Phys. Discuss.: 6 February 2008

Revised: 17 November 2008 - Accepted: 17 November 2008 - Published: 16 December 2008

\begin{abstract}
A method is presented that combines individual ground based ultraviolet (UV) measurements and satellite data from MSG (Meteosat Second Generation) to generate a UV-Index map all over the region of Austria. Cloud modification factors (CMFs) gathered from satellite images give an area wide information of the cloud situation but compared to ground based measurements significant discrepancies have been found. These discrepancies are especially large for high mountain stations but also for low altitude sites differences between satellite derived and ground based data in the order of $15 \%$ have been found. To overcome these discrepancies a correction procedure based on the correlation of both data sets at the pixel of the measurement stations is developed. The uncertainty of the final UV-Index map is evaluated exemplarily for the pixel of the measurement sites Bad Vöslau and München by eliminating the information of both sites from the UV-Index map generation process and comparing afterwards calculated and measured results. More than 5000 maps for all weather conditions have been evaluated leading to a mean agreement of 1.02 (standard deviation \pm 0.31 , Bad Vöslau) and 1.01 (standard deviation \pm 0.34 , München) between calculated and measured UV-Indexes.
\end{abstract}

\section{Introduction}

Since the discovery of the Antarctic ozone hole in 1985 several studies of stratospheric ozone depletion have been performed showing that this phenomenon is not only confined to the Antarctic continent. This fact led to concerns about increasing UV radiation at the earth's surface and motivated

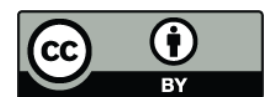

Correspondence to: B. Schallhart (barbara.schallhart@i-med.ac.at) more profound investigations in measuring and modelling UV radiation. A lot of countries including Austria established monitoring networks to quantify the amount of UV radiation at the ground with high accuracy. At the present time the Austrian UVB monitoring network consists of fifteen ground based detectors in selected locations. Since the main climatological parameters influencing UV radiation like clouds, ozone and aerosols vary on a small scale depending on geographical conditions and wind situation, it is difficult to extend the localized UV measurements to a broader scale without additional information. Although ground based UV detectors are the reference in terms of accuracy they only offer information of the incident solar radiation in single spots. A method to obtain radiation maps based on ground measurements only is to interpolate spatially between the single point measurements. By using the Kriging method (Krige, 1981) together with elevation maps and long term correlations between the measurements, simple radiation maps are obtained (Schmalwieser and Schauberger, 2001). A shortcoming of this method is that effects of changing clouds on the surface radiation can not be taken into account.

On the other hand satellite images provide area-wide information of the atmosphere and the surface. So methods have been developed to map the surface UV radiation by combining modelling and satellite data (Verdebout, 2000). Surface UV radiation maps of Greece generated by this method can be found at http://lap.physics.auth.gr/uvnet.gr/. However, determining cloud characteristics from satellite images is a difficult task resulting in substantial uncertainties in the gathered cloud information. Moreover a disadvantage of satellite data is due to the dependency of the accuracy on the pixel resolution. Regional variations of the atmosphere and changes in the surface are smoothed over some square kilometres.

Published by Copernicus Publications on behalf of the European Geosciences Union. 
Here we present a method that uses a combination of ground based measurements as well as satellite derived data to calculate an area-wide UV-Index map of Austria. On one hand this method benefits from high accuracy of ground measurements and on the other hand from high geographical coverage of the satellite data.

\section{Data from satellite and ground based measurements}

The algorithm is based on two independent data sets that are satellite images from MSG taken at three spectral channels in the visible and infrared and ground based measurements of UV radiation gathered by the Austrian UVB monitoring network.

\subsection{Satellite images from MSG}

Since April 2005 so-called High Rate SEVIRI Image Data (EUMETSAT, 2005) is collected. This data is measured with the SEVIRI (Spinning Enhanced Visible and Infra-Red Imager) radiometer onboard the satellites of the Meteosat Second Generation series operated by the European Organisation for the Exploitation of Meteorological Satellites (EUMETSAT). On a 15 min basis images of the full disk of the earth are received in eleven spectral channels. The spatial resolution of the subsatellite point is $3 \times 3 \mathrm{~km}$, in the region of Austria the pixel size is approximately $5.5 \times 4 \mathrm{~km}$ (northsouth $\times$ east-west) which corresponds to a resolution of $0.05^{\circ}$. For further analysis only images at $0.6 \mu \mathrm{m}, 1.6 \mu \mathrm{m}$ and $12 \mu \mathrm{m}$ are used.

\subsection{The Austrian UVB monitoring network}

The Austrian UVB monitoring network is operational since 1997 and consists of ten primary stations that are equipped with broadband UV-Biometers (Model 501 from Solar Light Co. Inc.) for measuring erythemally weighted solar UV irradiance. At the present time data from additional five measurement stations with broadband detectors (also measuring erythemally weighted solar UV irradiance) is received to improve the geographical coverage thereby improving the quality of the generated UV-Index map. Within the area of Austria twelve stations are distributed in order to cover the most populated areas as well as different levels of altitude, see Table 1. In addition two detectors situated in Germany nearby the Austrian border at München and at Zugspitze as well as one station located at Davos (Switzerland) contribute to the network. The location of all stations within the UV-Index map can be seen in Fig. 4.

International intercomparisons of broadband detectors (Leszczynski et al., 1998; Bais et al., 2000; Gröbner et al., 2008) have shown that there is a great variability between results from different instruments. Moreover the same detector may change from year to year by $\pm 10 \%$. Therefore the detectors are calibrated once a year by determining the spectral sensitivity in the laboratory. The absolute calibration is carried out by comparing solar broadband measurements to simultaneous measurements of a well-calibrated spectroradiometer. The uncertainty of the calibration is about $\pm 7 \%$ (Blumthaler, 2004). In addition quality control is permanently done by comparing the measurements to clear sky calculations carried out with the model FastRT (Engelsen and Kylling, 2005).

The UV measurements are recorded as mean values over 10 or $30 \mathrm{~min}$. Afterwards the raw data is sent to Innsbruck. To obtain absolute values the raw data is multiplied by a calibration function that is dependent on solar zenith angle and ozone. Finally the results are translated in units of the UVIndex. The UV-Index is defined by the World Health Organization (WHO, 2002) as the measured solar global irradiance (in $\mathrm{mW} \mathrm{m}^{-2} \mathrm{~nm}^{-1}$ ) weighted with the erythemal action spectrum of the human skin, integrated from 250 up to $400 \mathrm{~nm}$ and finally divided by $25 \mathrm{~mW} \mathrm{~m}^{-2}$. This leads to a dimensionless positive number that is ranging for most conditions in Europe from 0 to 10. Moreover at the high mountain station Sonnblick also the ozone column is measured daily by a Brewer spectroradiometer. This data is available at the following day. In order to calculate a clear sky UV prognosis of the current day ozone forecast data from DWD (Deutscher Wetter Dienst, German Weather Service) is used. Since one aim of the Austrian UVB Monitoring network is to increase the awareness of possible health risks induced by long-term exposure to solar UV radiation, all measurements and derived results including the UV-Index map are open to the public at http://www.uv-index.at/.

\section{Method}

First of all the two independent data sets are checked for consistency. Therefore satellite images as well as ground based measurements are evaluated in terms of cloud modification factors by using different algorithms. The cloud modification factor $F$ is defined as a scaling factor giving the reduction of the clear sky UV-Index $I_{\text {clear }}$ due to the presence of clouds, see Eq. (1)

$I_{\text {cloudy }}=I_{\text {clear }} \times F$

where $I_{\text {cloudy }}$ is the reduced UV-Index due to clouds and $F=0 \ldots 1$. So the lower limit of the cloud modification factor $F=0$ describes a totally overcast sky where no radiation is passing through the cloud field, whereas $F=1$ means that there are no clouds at all (clear sky).

\subsection{Cloud modification factors from satellite images}

The processor for retrieving the cloud modification factor from MSG is based on an algorithm previously developed 
Table 1. List of stations contributing to the Austrian UVB monitoring network. The detector type is either a Model 501 from Solar Light (SL501) or a UV-S-E-T from Scintec - Kipp \& Zonen. Moreover correlation coefficient, mean value and standard deviation $(k=1)$ of the ratio $F_{\mathrm{MSG}}$ to $F_{\text {ground }}$ are given.

\begin{tabular}{lccccccc}
\hline Stations & $\begin{array}{c}\text { Altitude } \\
{[\mathrm{m} \text { a.s.1. }]}\end{array}$ & $\begin{array}{c}\text { Latitude } \\
{\left[{ }^{\circ} \mathrm{N}\right]}\end{array}$ & $\begin{array}{c}\text { Longitude } \\
{\left[{ }^{\circ} \mathrm{E}\right]}\end{array}$ & $\begin{array}{c}\text { Detector } \\
\text { type }\end{array}$ & $\begin{array}{c}\text { Correlation } \\
\text { coefficient }\end{array}$ & $\begin{array}{c}\text { Mean } \\
\text { value }\end{array}$ & $\begin{array}{c}\text { Standard } \\
\text { deviation }\end{array}$ \\
\hline Wien & 153 & 48.26 & 16.43 & SL501 & 0.90 & 0.88 & 0.26 \\
Grossenzersdorf & 156 & 48.20 & 16.57 & UV-S-E-T & 0.86 & 0.89 & 0.28 \\
Bad Vöslau & 286 & 47.97 & 16.20 & SL501 & 0.86 & 0.89 & 0.29 \\
Steyregg/Linz & 335 & 48.29 & 14.35 & SL501 & 0.88 & 0.94 & 0.33 \\
Graz & 348 & 47.10 & 15.42 & SL501 & 0.89 & 0.94 & 0.29 \\
Dornbirn & 410 & 47.43 & 9.73 & SL501 & 0.87 & 0.86 & 0.30 \\
Klagenfurt & 448 & 46.65 & 14.32 & SL501 & 0.86 & 0.93 & 0.27 \\
München & 530 & 48.15 & 11.57 & UV-S-E-T & 0.87 & 0.84 & 0.28 \\
Innsbruck & 577 & 47.26 & 11.38 & SL501 & 0.80 & 0.84 & 0.29 \\
Mariapfarr & 1153 & 47.15 & 13.75 & SL501 & 0.81 & 0.82 & 0.28 \\
Kanzelhöhe & 1526 & 46.68 & 13.91 & SL501 & 0.82 & 0.89 & 0.31 \\
Davos & 1610 & 46.80 & 9.83 & SL501 & 0.60 & 0.66 & 0.28 \\
Hafelekar & 2275 & 47.32 & 11.39 & SL501 & 0.53 & 0.87 & 0.46 \\
Zugspitze & 2660 & 47.42 & 10.98 & UV-S-E-T & 0.61 & 0.77 & 0.38 \\
Sonnblick & 3106 & 47.05 & 12.96 & SL501 & 0.44 & 0.69 & 0.38 \\
\hline
\end{tabular}

for mapping the erythemal surface irradiance using METEOSAT/MVIRI images, satellite ozone and ancillary geophysical data (Verdebout, 2000). The surface erythemal dose rate is obtained by interpolation in a look up table (LUT) of modelled erythemal irradiance (CIE87 action spectrum), the entries of which are solar zenith angle, total column ozone amount, cloud optical thickness, near surface horizontal visibility, surface elevation and UV albedo. The LUT is generated from fully coupled radiative transfer calculations performed with the UVspec code of the libRadtran package (Mayer and Kylling, 2005). In order to allow using the cloud optical thickness retrieved by inversion from a single band image of MSG/SEVIRI the cloud model is constrained to be very simple. In practice, it is a single layer water cloud ( $1 \mathrm{~km}$ thick), $1 \mathrm{~km}$ above the surface and with a $7 \mu \mathrm{m}$ droplet radius. The optical thickness is modulated with the cloud density. All other atmospheric parameters are given standard values (i.e. US standard atmosphere, "tropospheric" aerosol model, background stratospheric aerosols). The surface reflectance is assumed to be Lambertian.

Both satellite and non-satellite (synoptic observations, digital elevation model) data are exploited to assign values to the influencing factors. The cloud modification factor $F_{\mathrm{MSG}}$ as defined in Eq. (1) is simply obtained by dividing the surface erythemal irradiance by what would be its value in the absence of clouds (obtained from the LUT with a cloud density set to 0$)$. As the radiative transfer calculations are onedimensional, the satellite-derived CMF never exceeds 1 . In other terms, CMFs larger than one, as sometimes produced by 3-D scattering effects within the cloud field structure, cannot be modelled. The CMF maps are generated on a latitude/longitude grid with a spatial resolution of $0.05^{\circ} \times 0.05^{\circ}$.
This resolution roughly corresponds to that of MSG over central Europe. An example of a CMF map for broken cloud conditions is given in Fig. 3.

In the original method, total column ozone is extracted from the gridded TOMS daily data or other ozone sensors data (e.g. TOVS, GOME, OMI) and the aerosols are taken into account by gridding daily measurements of near surface horizontal visibility performed at ground stations. For the application described here, the CMF is generated in near real time, within about $10 \mathrm{~min}$ of the MSG images acquisition. The above mentioned data on ozone and visibility are not available within this delay. Because the output is the CMF and not the UV-Index itself, it was judged that climatological values could be used instead. The daily climatological values for total column ozone and visibility were obtained by averaging the TOMS and gridded visibility data over the 1984 to 2003 period, with a 10 days running window. The averaging period corresponds to that of a 20 years data set of erythemal radiation maps over Europe, generated with METEOSAT/MVIRI, for which the data were readily available (Verdebout, 2004). The digital elevation model is derived from the GTOPO30 data set from United States Geological Survey (USGS) by arithmetically averaging the altitude in each $0.05^{\circ} \times 0.05^{\circ} \mathrm{CMF}$ output cell. The values for the last two influencing factors, i.e. cloud density and UV albedo are retrieved from MSG/SEVIRI images.

This is done using another LUT simulating the "at sensor radiance" (proportional to the image digital count), in the SEVIRI visible band centred at $0.6 \mu \mathrm{m}$. The entries of this second LUT are solar zenith angle, SEVIRI viewing zenith angle, relative azimuth between illumination and viewing vectors, effective surface albedo and cloud density (with the 

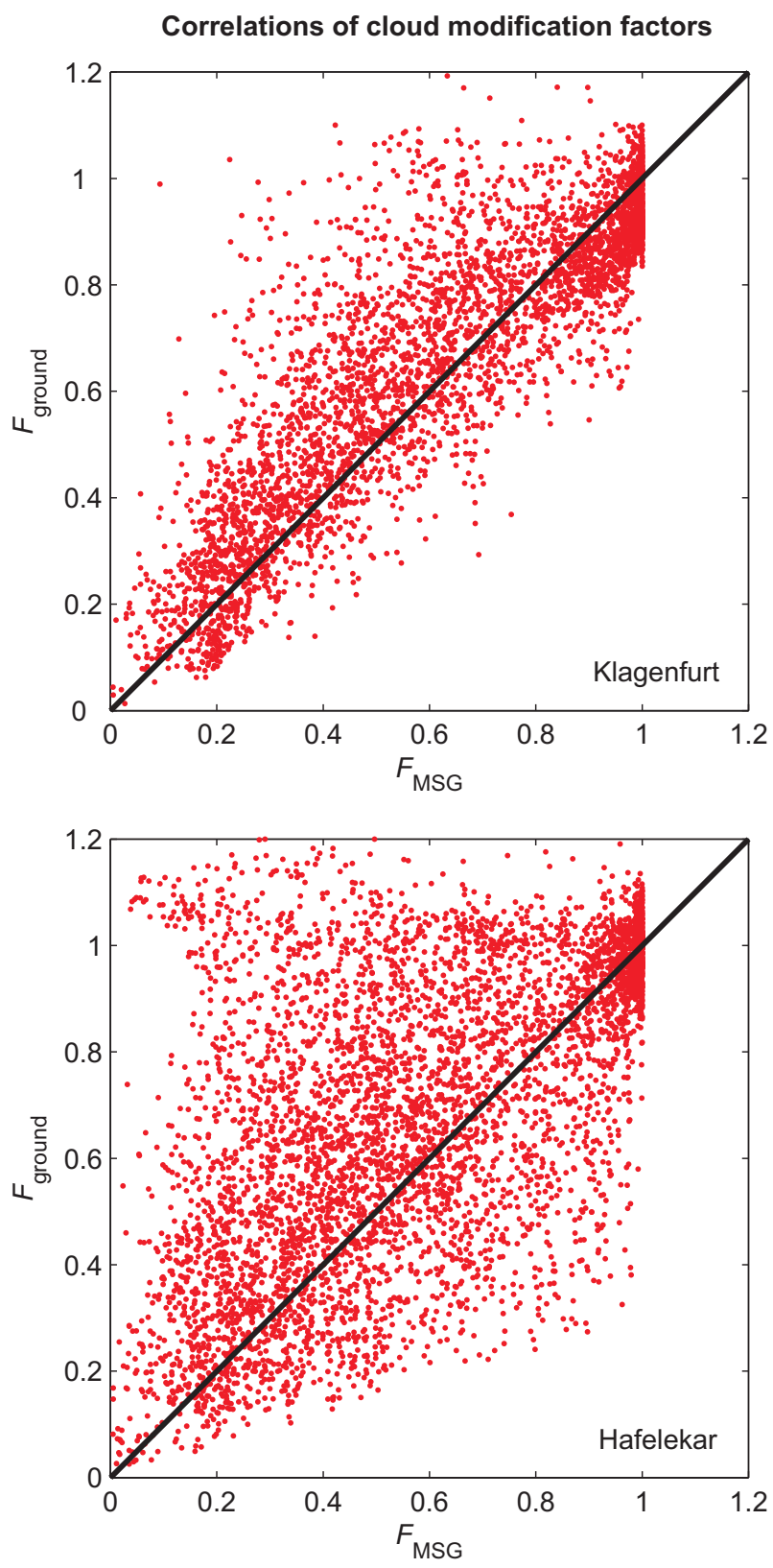

Fig. 1. Comparison of cloud modification factors estimated from satellite images $\left(F_{\mathrm{MSG}}\right)$ and obtained from ground based measurements $\left(F_{\text {ground }}\right)$ for two sites (Klagenfurt and Hafelekar) of the Austrian UVB monitoring network. The black line indicates perfect correlation. All the data from April 2005 up to November 2007 is included which sums up to 4311 (5315) data points for Klagenfurt (Hafelekar) gathered on 543 (670) days in this period.

same cloud model as for the UV LUT). A preliminary step consists in generating an effective surface albedo map by finding cloud free pixels in a series of ten SEVIRI images corresponding to the same time slot of ten consecutive days (the nine previous days are used). In most cases, the cloud- less pixel is chosen as the one corresponding to the lowest signal in the $0.6 \mu \mathrm{m}$ SEVIRI band. However, if the surface reflectance is high, the darkest signal does not necessarily indicate the absence of clouds (the snow reflectance can be higher than that of clouds). Therefore, if the minimal effective surface reflectance is found to be above a certain threshold, the discrimination is refined by also using the $1.6 \mu \mathrm{m}$ near infrared and the $12 \mu \mathrm{m}$ thermal infrared bands (Verdebout and Gröbner, 2004). These two bands add other discriminating criteria. At $1.6 \mu \mathrm{m}$, a water cloud appears darker than snow while the pixel brightness temperature (computed from $12 \mu \mathrm{m}$ band) will in general be higher when cloud free than when cloud covered, even if snow is present. These criteria together with the spatial context (i.e. the albedo values found at neighbouring pixels, the altitude) are used to make a decision on the day in the series that is chosen as cloud free (for each pixel). This decision process also uses histograms of the $1.6 \mu \mathrm{m}$ signal and of the brightness temperature, generated for a surrounding area, to dynamically set discriminating thresholds. Although the two additional bands help, the decision can still be wrong because ice clouds appear bright at $1.6 \mu \mathrm{m}$, because the surface can be warmer than the cloud top or simply because some areas are cloud covered during the 10 days. The snow covered areas are also a difficult case because the dynamic of the SEVIRI signal in the visible (with respect to cloud optical thickness) is much reduced. Especially in the area of the Austrian Alps this leads to discrepancies between satellite estimates and ground based measurements as described in Sect. 3.3.

Once the composite cloudless digital count image has been constructed, it is transformed in an effective albedo map by inversion, using the LUT reduced to the cloudless case. For each pixel, as values for all the other entries are known, the effective albedo map is then used to reduce the LUT to a single function yielding the $0.6 \mu \mathrm{m}$ SEVIRI signal dependence on the cloud density. The latter can then be retrieved by inversion from the $0.6 \mu \mathrm{m}$ SEVIRI signal for the day and time of interest.

The UV surface albedo is assigned uniform values for land $(0.03)$ and sea/ocean (0.06), except in the presence of snow. In this case it is given a value proportional to the $0.6 \mu \mathrm{m} \mathrm{SE}-$ VIRI band effective albedo. The rationale for proportionality between the albedos in the two spectral ranges is that partial snow cover should affect them in a similar way.

\subsection{Cloud modification factors from ground based mea- surements}

In order to obtain cloud modification factors for each site of the Austrian UVB monitoring network the measured UVIndexes $I$ have to be compared to site specific clear sky model calculations $\tilde{I}$ of the UV-Index. Then the CMF for each site $F_{\text {ground }}$ is obtained by

$$
F_{\text {ground }}=I / \tilde{I} \text {, }
$$


where $F_{\text {ground }}=0 \ldots 1.2$. The cloud modification factor can become greater than 1 (upper limit 1.2) because for clouds nearby the position of the sun the radiation passing through the atmosphere can be reflected at a cloud border thereby enhancing the radiation reaching the detector. In that case the measured value is greater than the clear sky model value. The enhancement of the measured value is allowed to become up to $20 \%$ of the model calculation result. For higher discrepancies a problem during the measurement is assumed and the data is discarded. The accuracy of the results of Eq. (2) is dependent on how representative the model calculations are for each site. This depends on the model used and on the chosen input parameters.

FastRT is a fast simulation tool for UV radiation developed by Ola Engelsen. Surface UV irradiances are obtained by interpolation in lookup tables of transmittances and reflectances. The lookup tables are computed using the accurate libRadtran atmospheric radiative transfer software package (Mayer and Kylling, 2005) with a couple of predefined input parameters. The agreement of both models when comparing calculated UV-Indexes for cloudless but aerosol loaded atmospheric conditions is at the 3\% level (Engelsen and Kylling, 2005). For the benefit of fast computational time FastRT is used although the set of variable input parameters is restricted. The input parameters are altitude, solar zenith angle, day of year, albedo, ozone (altitude dependent forecast from DWD) and Ångström coefficient beta (Ångström coefficient alpha=1.3 predefined). Albedo and Ångström coefficient beta are dependent on altitude and on the snow line. To obtain suitable clear sky model results for each measurement site reasonable parameters are chosen. To check measured and modelled UV-Index for consistency, measurements performed at clear sky conditions have to be identified. This is done by using the cloud modification factors of the satellite images. A cloud modification factor larger than 0.98 at a site pixel indicates clear sky conditions for the given time. Using two years of measured data fulfilling this criterion a mean value of 0.92 of the ratio measurement/model was found. So the modelled values are on average $8 \%$ higher than the measurements at all sites. Since no measurements of albedo and aerosols are available the estimated input parameters may lead to this offset. The ratio measurement/model is also varying from day to day which indicates changing atmospheric conditions with time. To reduce the error in Eq. (2) that is caused by this inconsistency, every day clear sky measurements and model results are compared leading to a correction factor for all modelled values. If there are no clear sky measurements on a given day the default value of 0.92 is used to reduce the model results.

\subsection{Correlation of ground based and satellite derived CMF}

Statistical numbers showing the correlation between cloud modification factors estimated from satellite images and calculated from ground based measurements are given in Ta- ble 1. For this analysis data points from April 2005 up to November 2007 with a maximal time delay of $\pm 7.5 \mathrm{~min}$ have been chosen. This corresponds to the maximum delay between satellite image (every $15 \mathrm{~min}$ ) and the center of the measurement interval (10 or $30 \mathrm{~min}$ ) of the ground based devices.

The correlation between both quantities is around 0.88 for low altitude sites and becomes even worse for the high mountain stations. Exemplarily Klagenfurt (448 m a.s.l) and Hafelekar (2275 ma.s.l) are chosen to show the correlation between both cloud modification factors in Fig. 1. For Hafelekar (Klagenfurt) 5315 (4311) time matching (maximal time delay $\pm 7.5 \mathrm{~min}$ ) data points for all weather conditions have been found from April 2005 up to November 2007. The black line indicates perfect correlation. The correlation for Klagenfurt data is best for clear sky conditions (around [1,1]) and overcast sky (below [0.2, 0.2]) but overall the scattering is quite bad. For the high mountain station Hafelekar the results are even worse, which is partly due to the problem of snow detection from satellite images described above. For clear sky conditions and snow covered mountains $\left(F_{\text {ground }} \rightarrow 1\right.$ ) satellite derived cloud modification factors $\left(F_{\mathrm{MSG}}\right)$ are often low indicating clouds instead of snow. Moreover the low resolution of satellite images is more critical when compared with single spot measurements in mountainous regions (Hafelekar) than in flat country (Klagenfurt). Since these results are not satisfying correction methods had to be developed.

3.4 Correction of satellite derived cloud modification factors

For the final calculation of the UV-Index map area-wide cloud modification factors are needed, so the satellite estimates have to be corrected. Since the determination of cloud modification factors for snow covered satellite pixels is inaccurate, all CMF pixels are split up in two groups with altitudes below and above the snow line. Unfortunately there is no data of the distribution of the snow line available in Austria. So the snow line altitude is estimated daily by observation and assumed constant over the whole area. Based on the correlation of CMFs at the location of the ground site, a correction function is determined and spatially expanded to a region of $4^{\circ}$ around the site with decreasing effect. Since the final UV-Index map covers the area from $46.25^{\circ}$ up to $49.2^{\circ}$ in latitude and from $9.3^{\circ}$ up to $17.3^{\circ}$ in longitude, all pixels lie within the correction range of at least one site pixel. There are different correction functions dependent on the correlation of CMFs at each site and dependent on the site altitude compared to the snow line.

Case 1: $F_{\text {ground }}>0.9$ and $F_{\mathrm{MSG}}>0.9$ and altitude below the snow line: In this case there is a good correlation between both CMFs and the correction function $\operatorname{corr}\left(F_{\mathrm{MSG}}\right)$ is the identity, see Fig. 2, Case 1. So the corrected CMF pixel 


\section{Correction functions}
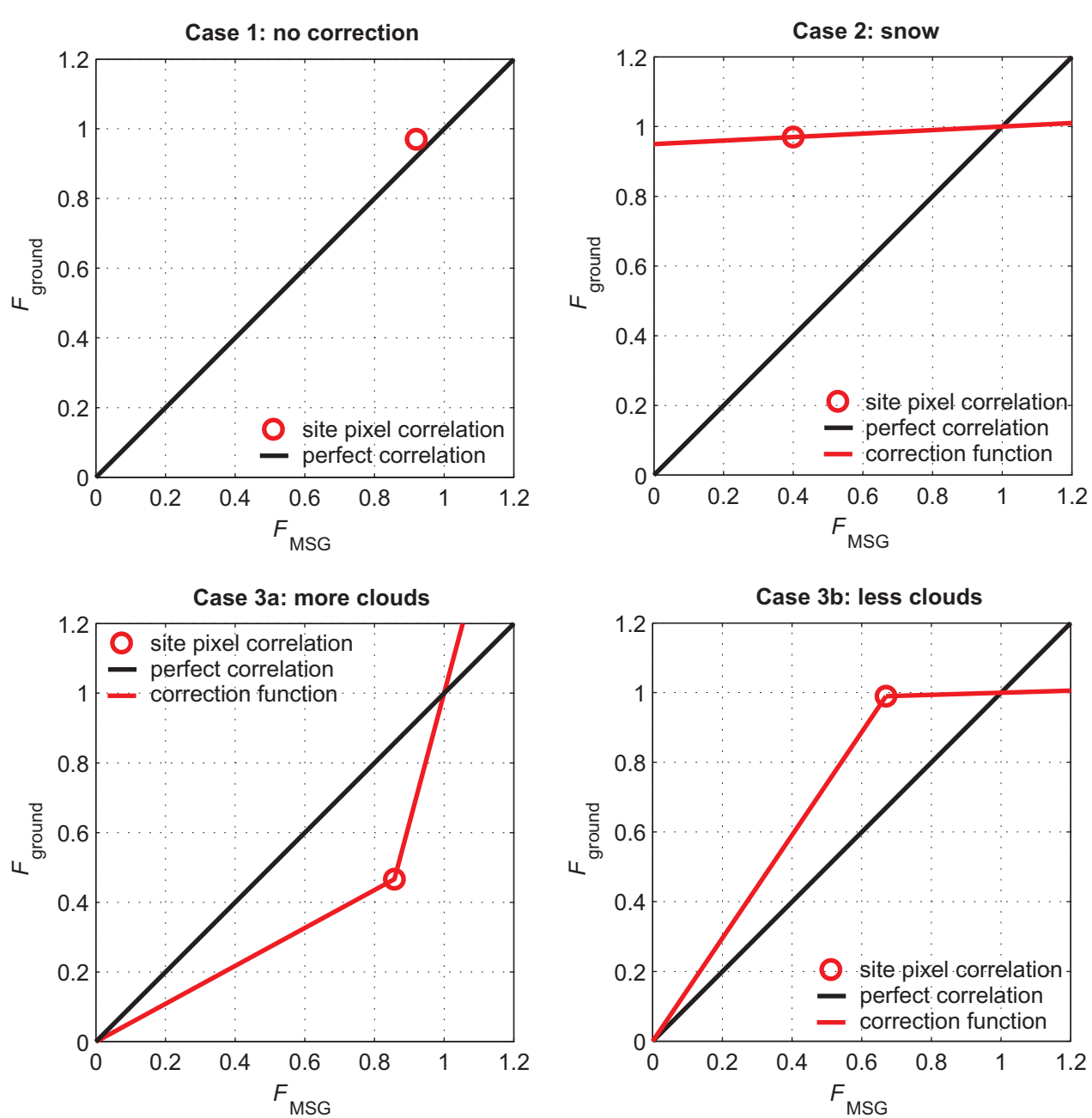

Fig. 2. Correction functions dependent on the site pixel correlation of CMFs and on the altitude in relation to the snow line as described in detail in Sect. 3.4.

$F_{\mathrm{MSG}}^{\mathrm{corr}}(x)$ is defined as

$F_{\mathrm{MSG}}^{\mathrm{corr}}(x)=F_{\mathrm{MSG}}(x)$

with $x=0^{\circ} \ldots 4^{\circ}$.

Case 2: $\quad F_{\text {ground }}>0.8$ and altitude above the snow line: There can be the problem of snow being detected as clouds in the vicinity of this site. This would lead to pixels with too low UV-Indexes. Since the final UV-Index maps are open to the public to inform about potential health risks, it is better to obtain bigger values for the UV-Index than lower ones. Therefore this correction is made even if there is a good correlation between $F_{\text {ground }}$ and $F_{\mathrm{MSG}}$.

The correction function $\operatorname{corr}\left(F_{\mathrm{MSG}}\right)$ is given by a straight line trough the points $\left[F_{\mathrm{MSG}}, F_{\text {ground }}\right]$ and $[1,1]$, see Fig. 2, Case 2. With this correction function all satellite determined CMFs with an altitude above the snow line are increased lin- early with decreasing distance to the site pixel in the following way:

$F_{\mathrm{MSG}}^{\mathrm{corr}}(x)=\operatorname{corr}\left(F_{\mathrm{MSG}}(x)\right)-\frac{x}{4}\left[\operatorname{corr}\left(F_{\mathrm{MSG}}(x)\right)-F_{\mathrm{MSG}}(x)\right]$

with $x=0^{\circ} \ldots 4^{\circ}$.

Case 3: Remaining correlations ( $F_{\text {ground }} \leq 0.9$ and $\left.F_{\mathrm{MSG}} \leq 0.9\right)$ with altitudes below the snow line and with altitudes above the snow line $\left(F_{\text {ground }} \leq 0.8\right)$ : The correction function is a combination of two straight lines. The first one is starting at $[0,0]$ and ending at $\left[F_{\mathrm{MSG}}, F_{\text {ground }}\right]$ whereas the second one is from $\left[F_{\mathrm{MSG}}, F_{\text {ground }}\right]$ to $[1,1]$, see Fig. 2 , Cases $3 \mathrm{a}$ and $3 \mathrm{~b}$. Again the correction is implemented as defined in Eq. (4) where the effect is decreasing with increasing distance. In this case the corrected CMF can be higher or lower than the satellite estimate taking into account situations with less or more clouds. 
The correction methods described above strongly depend on the correlation of CMFs between satellite estimate and ground measurement at a single site. Since most CMF pixels are in between $4^{\circ}$ of at least two site pixels, the distance weighted corrections gained from the two closest site pixels are finally used to calculate the corrected CMF value $\widehat{F}_{\text {MSG }}^{\text {corr }}(i)$ for pixel $i$, as defined in Eq. (5)

$\widehat{F}_{\mathrm{MSG}}^{\mathrm{corr}}(i)=\frac{F_{\mathrm{MSG}}^{\mathrm{corr}}\left(x_{1}\right) \times x_{2}^{2}+F_{\mathrm{MSG}}^{\mathrm{corr}}\left(x_{2}\right) \times x_{1}^{2}}{x_{1}^{2}+x_{2}^{2}}$.

The distances from pixel $i$ to the two closest site pixels are denoted with $x_{1}$ and $x_{2}$. Because $F_{\text {ground }} \leq 1.2$ the correction can lead to $\widehat{F}_{\text {MSG }}^{\text {corr }}>1$ in rare cases. So also $\widehat{F}_{\text {MSG }}^{\text {corr }}$ is limited to 1.2 .

An example of the effect of the correction method can be seen in Fig. 3. First the cloud modification factor map estimated from satellite images that were obtained on 2 September 2007 at 16:30 UTC is shown (first row). In the second row the corrected CMF map is given and in the third row the difference of both maps can be seen. The snow line on this day was at an altitude of $2800 \mathrm{~m}$ a.s.l. which means that there was no snow except in the glacier regions. So all stations except the high mountain station Sonnblick were below the snow line. Measurements at Zugspitze and in Mariapfarr lead to a reduction in $\widehat{F}_{\text {MSG }}^{\text {corr }}$ whereas measurements in Innsbruck and at Hafelekar cause an enhancement. Moreover the decreasing effect of the correction with increasing distance can be seen nicely.

\subsection{UV-Index map}

In a final step the UV-Index map is calculated. Therefore a lookup table of clear sky UV-Indexes is computed with FastRt. The input parameters are altitudes in steps of $200 \mathrm{~m}$ from 0 to $4000 \mathrm{~m}$, solar zenith angle, day of year, albedo, ozone (altitude dependent forecast from DWD) and Ångström coefficient beta. Albedo and Ångström coefficient beta are dependent on altitude and on the snow line. The solar zenith angle is calculated for the central pixel of the map at the time given by the satellite images. As described in Sect. 3.2 a correction factor is applied to the modelled values to overcome discrepancies between model and clear sky measurements.

Next the corrected cloud modification factor map $\widehat{F}_{\text {MSG }}^{\text {corr }}$ is interpolated to the grid of the topography map with a resolution of approximately $1 \times 1 \mathrm{~km}$. Then for each pixel of the topography map the clear sky UV-Index is obtained from the lookup table. In the end the UV-Index map is generated by scaling the clear sky UV-Index with the corrected CMF for each pixel. The calculated UV-Index map for 12:45 local time on 16 August 2007 is shown in Fig. 4. The structure of the UV-Index which is indicated by predefined colors is caused by the satellite data derived cloud field and by topography. Based on new sets of satellite im-
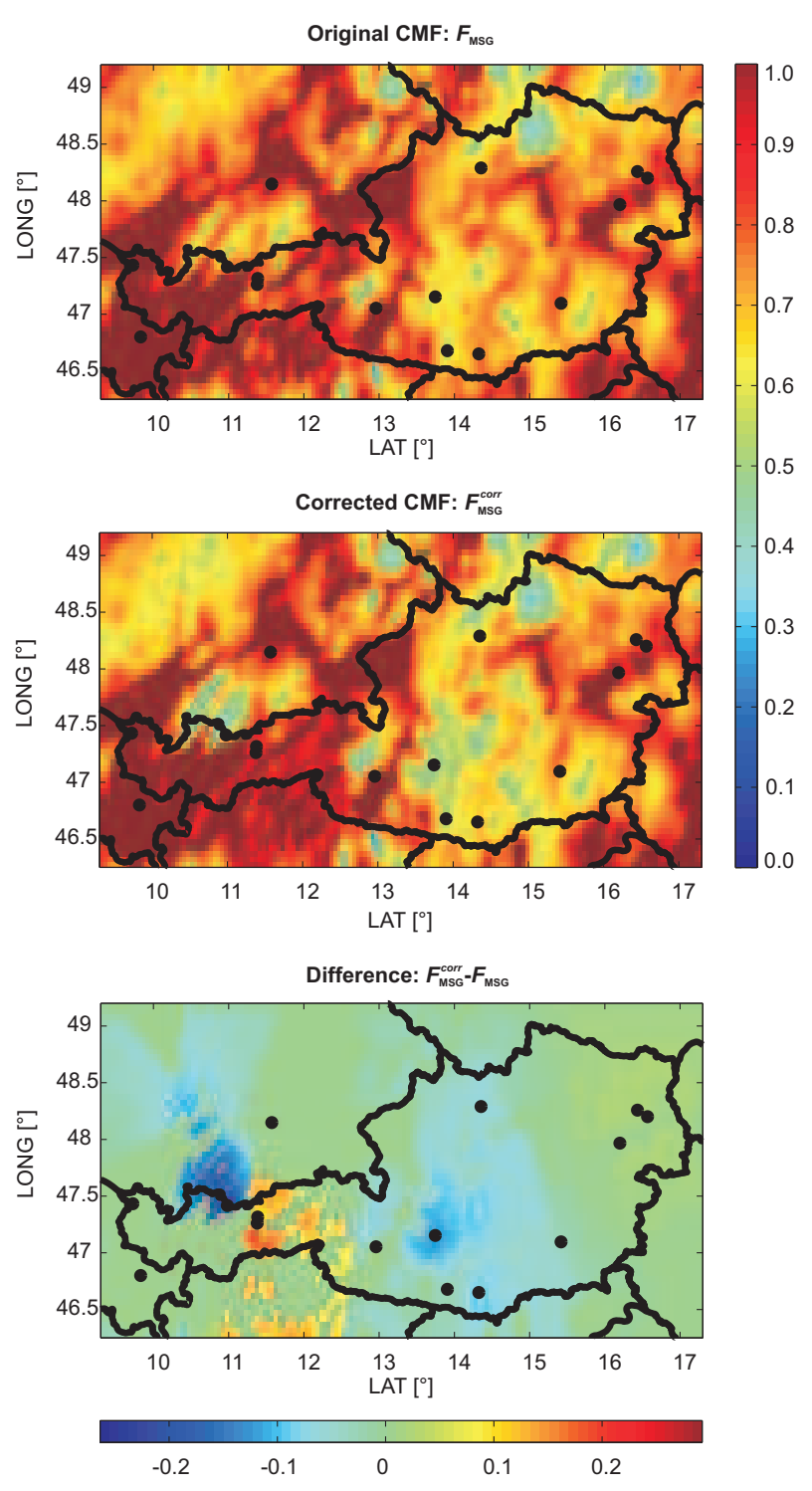

Fig. 3. Cloud modification factor map estimated from satellite images (first row) and cloud modification factor map corrected by means of ground based measurements (second row). The effect of the correction method is demonstrated as difference of both maps (third row), showing regions of enhancement and reduction in CMF. Black dots indicate the position of the ground based measurement sites of the Austrian UVB monitoring network.

ages every 15 min UV-Index maps are generated and available at http://www.uv-index.at/ with a time delay smaller than $30 \mathrm{~min}$.

\subsection{Accuracy of the method}

The UV-Index map is obtained by multiplication of clear sky model calculations with cloud modification factors determined from satellite images. Since the model calculations 


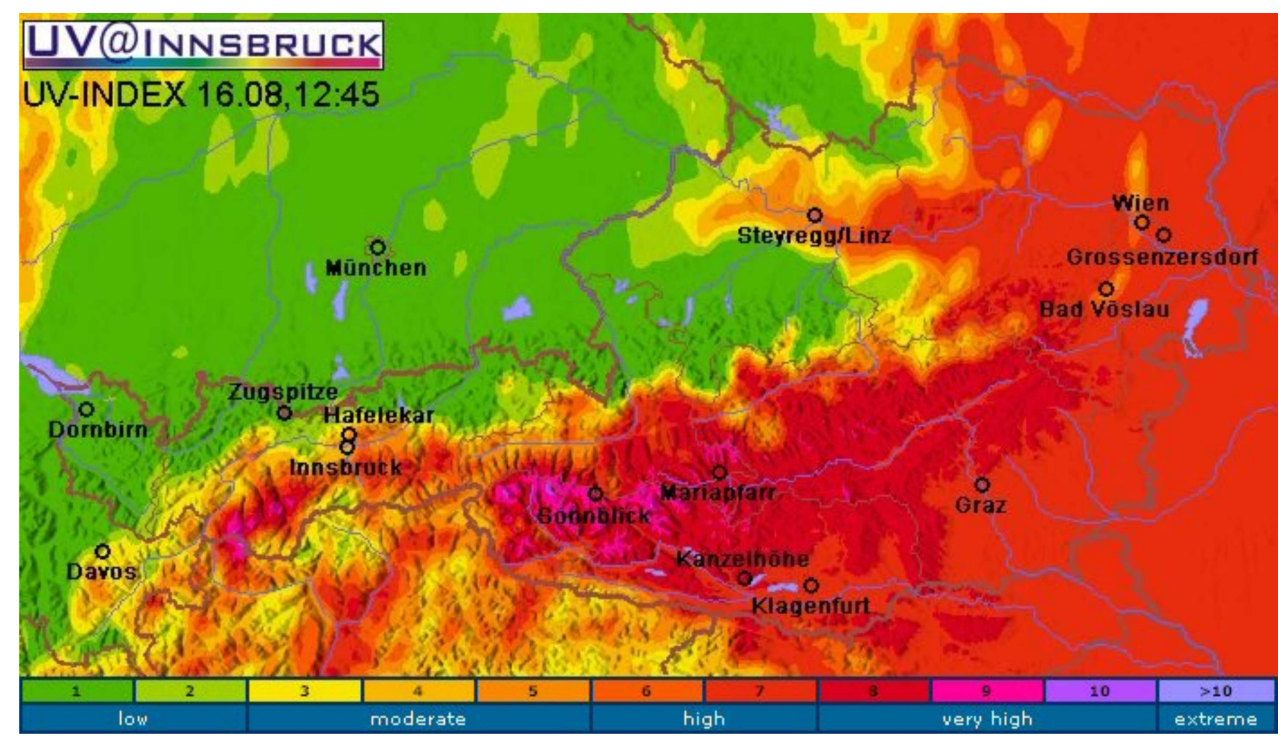

Fig. 4. UV-Index map generated by means of ground based UV measurements and cloud information derived from MSG satellite images on 16 August 2007 at 12:45 local time. The UV-Index is indicated by predefined colors.

are scaled to fit the clear sky measurements their uncertainty should be relatively small. The main part of the uncertainty in the UV-Index stems from erroneous CMFs.

To determine cloud modification factors from satellite images $\left(F_{\mathrm{MSG}}\right)$ in near real time is a difficult process yielding relatively large uncertainties. This is demonstrated by comparing CMFs determined from ground based measurements $F_{\text {ground }}$ with $F_{\text {MSG }}$ for all weather conditions, see Table 1. The mean value of the ratio $F_{\mathrm{MSG}}$ to $F_{\text {ground }}$ is around 0.86 for low altitude sites and becomes even lower for high altitude stations. Also the standard deviation $(k=1)$ is increasing from 0.3 (low altitudes) to 0.4 (high altitudes). To reduce the error in $F_{\mathrm{MSG}}$ a correction procedure taking into account ground based measurements is implemented as described above.

In the worst case no data of ground based measurements is available. Then the UV-Index map is calculated by scaling the uncorrected $F_{\mathrm{MSG}}$ with clear sky model calculations $I_{\text {clear }}$, see Eq. (1). Only then the uncertainty in the UV-Index $I_{\text {cloudy }}^{\mathrm{MSG}}$ becomes maximal.

On the other hand the accurate UV-Index at the pixel of the ground stations $I_{\text {cloudy }}^{\text {ground }}$ can be computed via Eq. (1) by using the CMFs derived from ground based measurements. By comparing both quantities $I_{\text {cloudy }}^{\text {MSG }}$ and $I_{\text {cloudy }}^{\text {ground }}$ at the pixel of the measurement stations the uncertainty in $I_{\text {cloudy }}^{\mathrm{MSG}}$ can be estimated. This comparison is simplified to an analysis of the ratio $F_{\text {ground }}$ to $F_{\mathrm{MSG}}$ by eliminating the clear sky model calculations $I_{\text {clear. }}$. Therefore the statistical numbers given in Table 1 are an estimation of the uncertainty in the calculated UV-Index when using uncorrected satellite derived CMFs.
As an example for Bad Vöslau this means a mean agreement of 0.89 with a standard deviation of $\pm 0.29(k=1)$, see Table 1 .

This large uncertainty is reduced by applying to $F_{\mathrm{MSG}}$ the correction procedure as described in Sect. 3.4, thereby also reducing the uncertainty in the final UV-Index map. Each pixel of the satellite derived CMF map is corrected by the help of ground based measurements stemming from the two neighbouring measurement sites. This correction method leads to a perfect correction at the pixel of the measurement stations, i.e. the corrected $F_{\mathrm{MSG}}$ matches $F_{\text {ground }}$ at the pixel of the stations. As a direct validation of the uncertainty in the final UV-Index the information of the station Bad Vöslau was removed from the analysis. So $F_{\mathrm{MSG}}$ at the pixel of Bad Vöslau was corrected based on the measurements of the nearby sites Wien and Grossenzersdorf. Then the estimated UV-Index $I_{\text {cloudy }}^{\mathrm{MSG}}$ as result of the algorithm at the pixel of Bad Vöslau was compared with the actual measurement $I_{\text {cloudy }}^{\text {ground }}$. In total 5196 maps from April 2005 up to November 2007 including all weather conditions were used for this test leading to a mean agreement of the ratio $I_{\text {cloudy }}^{\mathrm{MSG}} I_{\text {cloudy }}^{\text {ground }}$ of 1.02 with a standard deviation of $\pm 0.31(k=1)$. This is much better than the uncertainty when using uncorrected CMFs, see Table 1. As a second example 5499 maps (from April 2005 up to November 2007) were calculated without the information of the site München. The neighbouring measurement stations of München are not that close compared to the case of Bad Vöslau. Nevertheless the mean agreement of the ratio $I_{\text {cloudy }}^{\mathrm{MSG}}$ to $I_{\text {cloudy }}^{\text {ground }}$ for the München pixel was found to be 1.01 with a standard deviation of $\pm 0.34(k=1)$. This is again a considerable improvement compared to a mean agreement 
of 0.84 of the ratio $F_{\mathrm{MSG}}$ to $F_{\text {ground }}$ for the station München when using uncorrected CMFs (see Table 1).

These examples demonstrate the efficiency of the presented correction procedure and the importance of ground based measurements for the validation of satellite derived data.

\section{Conclusions}

A method to generate UV-Index maps in near real time over the region of Austria is presented. By combining satellite data and ground based UV measurements the main climatological parameters like clouds, ozone and aerosols are taken into account. Cloud modification factors gathered from satellite images give an area wide information of the cloud situation but compared to ground based measurements significant discrepancies have been found. These discrepancies are especially large for high mountain stations because the determination of CMFs from satellite images taken over snow covered mountains is a difficult task leading to large uncertainties. But also for low altitude sites differences between satellite derived and ground based data in the order of $15 \%$ have been found. To harmonise satellite derived and ground based data a correction procedure based on the correlation of both data sets at the pixel of the measurement stations was developed. The uncertainty of the final UV-Index map was evaluated exemplarily for the pixel of the measurement sites Bad Vöslau and München leading to an excellent mean agreement between calculated and measured UV-Indexes. As the data set of satellite images is expanding every day, more investigations in the site pixel correlation of cloud modification factors are planned thereby improving the correction methods.

Acknowledgements. The Austrian UVB monitoring network is funded by the Austrian Federal Ministry of Agriculture, Forestry, Environment and Water Management. We thank all contributors for their supply of data to the Austrian UVB monitoring network.

Edited by: J. Groebner

\section{References}

Bais, A., Topaloglou, C., Kazadtzis, S., Blumthaler, M., Schreder, J., Schmalwieser, A., Henriques, D., and Janouch, M.: Report of the LAP/COST/WMO intercomparison of erythemal radiometers, World Meteorological Organization-Global Atmosphere Watch, Geneva, Switzerland, Rep. No. 141, 2000.

Blumthaler, M.: Quality assurance and quality control methodologies within the Austrian UV monitoring network, Rad. Prot. Dos., 111(4), 359-362, 2004.

Engelsen O. and Kylling A.: Fast simulation tool for ultraviolet radiation at the Earth's surface, Opt. Eng., 44(4), 041012, doi:10.1117/12.639087, 2005.

EUMETSAT: MSG Ground Segment LRIT/HRIT Mission Specific Implementation, EUM/MSG/SPE/057, 5, 2005.

Gröbner, J., Hülsen, G., Vuilleumier, G., Blumthaler, M., Vilaplana, J. M., Walker, D., and Gil, J. E.: Report of the PMOD/WRC-COST calibration and intercomparison of erythemal radiometers, ftp.pmodwrc.ch/pub/publications/PMOD_ COST726_BBreport.pdf, 2008.

Krige, D. G.: Lognormal-de Wijsian Geostatistics for Ore Evaluation, South African Institute of Mining and Metallurgy Monograph Series, Geostatistics, 1, 51 pp., 1981.

Leszczynski, K., Jokela, K., Ylianttila, L., Visuri, R., and Blumthaler, M.: Erythemally weighted radiometers in solar UV monitoring: results from MWO/STUK intercomparison, Photochem. Photobiol., 67, 212-221, 1998.

Mayer, B. and Kylling, A.: Technical note: The libRadtran software package for radiative transfer calculations - description and examples of use, Atmos. Chem. Phys., 5, 1855-1877, 2005, http://www.atmos-chem-phys.net/5/1855/2005/.

Schmalwieser, A. W. and Schauberger, G.: A monitoring network for erythemally-effective solar ultraviolet radiation in Austria: determination of the measuring sites and visualisation of the spatial distribution, Theor. Appl. Climatol., 69(3), 221-229, doi:10.1007/s007040170027, 2001.

Verdebout, J.: A method to generate surface UV radiation maps over Europe using GOME, Meteosat, and ancillary geophysical data, J. Geophys. Res., 105(D4), 5049-5058, 2000.

Verdebout, J.: A European satellite-derived UV climatology available for impact studies, Radiat. Prot. Dosim., 111(4), 407-411, doi:10.1093/rpd/nch063, 2004.

Verdebout, J. and Gröbner, J.: Mapping natural surface UV radiation with MSG: first maps and comparison with METEOSAT derived results and reference measurements, in: Proceedings of the EUMETSAT Meteorological Satellite Conference, Prague, Czech Republic, 31 May-4 June 2004, 558 pp., 2004

World Health Organization: Global Solar UV Index: A Practical Guide, WHO, ISBN 924159007 6, Geneva, 2002. 